\title{
A Lower Bound on the Average-Case Complexity of Shellsort
}

\author{
Tao Jiang * $\quad$ Ming $\mathrm{Li}^{\dagger} \quad$ Paul Vitányi ${ }^{\ddagger}$
}

June 7, 2021

\begin{abstract}
We demonstrate an $\Omega\left(p n^{1+1 / p}\right)$ lower bound on the average-case running time (uniform distribution) of $p$-pass Shellsort. This is the first nontrivial general lower bound for average-case Shellsort.

Additional Key Words and Phrases: Sorting, Shellsort, computational complexity, average-case complexity, Kolmogorov complexity.
\end{abstract}

\section{Introduction}

The question of a nontrivial general lower bound (or upper bound) on the average complexity of Shellsort (due to D.L. Shell [12]) has been open for about four decades [4, 11]. We present such a lower bound for $p$-pass Shellsort for every $p$.

Shellsort sorts a list of $n$ elements in $p$ passes using a sequence of increments $h_{1}, \ldots, h_{p}$. In the $k$ th pass the main list is divided in $h_{k}$ separate sublists of length $\left\lceil n / h_{k}\right\rceil$, where the $i$ th sublist consists of the elements at positions $j$, where $j \bmod h_{k}=i-1$, of the main list $\left(i=1, \ldots, h_{k}\right)$. Every sublist is sorted using a straightforward insertion sort. The efficiency of the method is governed by the number of passes $p$ and the selected increment sequence $h_{1}, \ldots, h_{p}$ with $h_{p}=1$ to ensure sortedness of the final list. The original $\log n$-pass ${ }^{1}$ increment sequence $\lfloor n / 2\rfloor,\lfloor n / 4\rfloor, \ldots, 1$ of Shell [12] uses worst case $\Theta\left(n^{2}\right)$ time, but Papernov and Stasevitch [7] showed that another related sequence uses $O\left(n^{3 / 2}\right)$, and Pratt [9] extended this to a class of all nearly geometric increment sequences and proved this bound was tight. The currently best asymptotic method was found by Pratt [9]. It uses all $\log ^{2} n$ increments of the form $2^{i} 3^{j}<\lfloor n / 2\rfloor$ to obtain time $O\left(n \log ^{2} n\right)$ in the worst case. Moreover, since every pass takes at least $n$ steps, the average complexity using Pratt's increment sequence is $\Theta\left(n \log ^{2} n\right)$. Incerpi and Sedgewick [1] constructed a family of increment sequences for which Shellsort runs in $O\left(n^{1+\epsilon / \sqrt{\log n}}\right)$ time using $\left(8 / \epsilon^{2}\right) \log n$ passes, for every $\epsilon>0$. B. Chazelle (attribution in [10]) obtained the same result by generalizing Pratt's method: instead of using 2 and 3 to construct the increment sequence use $a$ and $(a+1)$ for fixed $a$ which yields a worst-case running time of $n \log ^{2} n\left(a^{2} / \ln ^{2} a\right)$ which is $O\left(n^{1+\epsilon / \sqrt{\log n}}\right)$ for $\ln ^{2} a=O(\log n)$. Plaxton, Poonen and Suel [8] proved an $\Omega\left(n^{1+\epsilon / \sqrt{p}}\right)$ lower bound for $p$ passes of Shellsort using any increment sequence, for some $\epsilon>0$; taking $p=\Omega(\log n)$ shows that the Incerpi-Sedgewick / Chazelle bounds are optimal for small $p$ and taking $p$ slightly larger shows a $\Theta\left(n \log ^{2} n /(\log \log n)^{2}\right)$ lower bound on the worst case complexity of Shellsort. Since every pass takes at least $n$ steps this shows an $\Omega\left(n \log ^{2} n /(\log \log n)^{2}\right)$ lower bound on the worst-case of every Shellsort increment sequence. For the average-case running time Knuth [4] showed $\Theta\left(n^{5 / 3}\right)$ for the best choice of increments in $p=2$ passes; Yao [13] analyzed the average case for $p=3$ but did not obtain a simple analytic form; Yao's analysis was improved by Janson and Knuth [2] who showed $O\left(n^{23 / 15}\right)$ averagecase running time for a particular choice of increments in $p=3$ passes. Apart from this no nontrivial results 2 are known for the average case; see $[4,10,11]$.

${ }^{*}$ Department of Computer Science, University of California, Riverside, CA 92521. Email: jiang@cs.ucr.edu. Supported in part by NSERC and CITO grants, and a UCR startup grant.

${ }^{\dagger}$ Dept of Computer Science, University of California, Santa Barbara, CA 93106, USA (on leave from the University of Waterloo). Email: mli@cs.ucsb.edu. Supported in part by NSERC and CITO grants and Steacie Fellowship.

${ }^{\ddagger}$ CWI, Kruislaan 413, 1098 SJ Amsterdam, The Netherlands. Email: paulv@cwi.nl. Supported in part by the EU via NeuroCOLT II Working Group and the QAIP Project.

1 "log" denotes the binary logarithm and "ln" denotes the natural logarithm.

${ }^{2}$ The trivial lower bound is $p n$ comparisons since every element needs to be compared at least once in every pass. 
Results: We show the result given in the abstract, more precisely, Theorem 2 . The main result is Theorem 1 below. This is the first advance on the problem of determining general nontrivial bounds on the average-case running time of Shellsort $[9,4,13,1,8,10,11]$. The proof was originally obtained using Kolmogorov complexity (for Kolmogorov complexity see [5]). The idea is to consider an "individually random" permutation of the input list (a permutation incompressible in the sense of Kolmogorov complexity). If one encodes every move of Shellsort cheaply, and if the algorithm does not make a certain number of moves, then one obtains a too short encoding of the random permutation - contradicting the incompressibility of it. ${ }^{3}$ Moreover, since the overwhelming majority of permutations is incompressible we obtain the bound on the average. It turns out that the argument can be translated to a counting argument. This we have done and present only the more elementary and shorter counting argument here. The original proof using Kolmogorov complexity is given in the preliminary version [6]. It is instructive that thinking in terms of code length and Kolmogorov complexity enabled advances in this problem.

\section{The Lower Bound}

A Shellsort computation consists of a sequence of comparison and inversion (swapping) operations. We count just the total number of data movements (here inversions) executed. The lower bound obtained below holds a fortiori for the number of comparisons. The proof is based on the following intuitive idea: There are $n$ ! different permutations. Given the sorting process (the insertion paths in the right order) one can recover the correct permutation from the sorted list. Hence one requires $n$ ! pairwise different sorting processes. This gives a lower bound on the minimum of the maximal length of a process.

Theorem 1 Let $0<\epsilon<1$ and $n, p$ satisfy $p \leq(\epsilon \log n) / \log e$. For every p-pass Shellsort algorithm and every increment sequence, every subset of $n ! / 2^{n}$ input permutations of $n$ keys contains an input permutation that uses $\Omega\left(p n^{1+(1-\epsilon) / p}\right)$ inversions (and comparisons).

Proof. Let the list to be sorted consist of a permutation $\pi$ of the elements $1, \ldots, n$. Consider a $\left(h_{1}, \ldots, h_{p}\right)$ Shellsort algorithm $A$ where $h_{k}$ is the increment in the $k$ th pass and $h_{p}=1$. For any $1 \leq i \leq n$ and $1 \leq k \leq p$, let $m_{i, k}$ be the number of elements in the $h_{k}$-chain containing element $i$ that are to the left of $i$ at the beginning of pass $k$ and are larger than $i$. Observe that $\sum_{i=1}^{n} m_{i, k}$ is the number of inversions in the initial permutation of pass $k$, and that the insertion sort in pass $k$ requires precisely $\sum_{i=1}^{n}\left(m_{i, k}+1\right)$ comparisons. Let $N$ denote the total number of inversions:

$$
N:=\sum_{k=1}^{p} \sum_{i=1}^{n} m_{i, k} .
$$

Claim 1 Given all the $m_{i, k}$ 's in an appropriate fixed order, we can reconstruct the original permutation $\pi$.

Proof. The $m_{i, p}$ 's trivially specify the initial permutation of pass $p$. In general, given the $m_{i, k}$ 's and the final permutation of pass $k$, we can reconstruct the initial permutation of pass $k$.

Therefore, to every input permutation there must correspond a unique combination of $N$ together with appropriate fixed order (say in lexicographical order of subscripts) of elements of a partition as in (1). How many such partitions are there? Choosing $a$ elements out of an ordered list of $a+b$ elements divides the remainder into a sequence of $a+1$ possibly empty sublists. Hence there are

$$
D(N):=\left(\begin{array}{c}
N+n p-1 \\
n p-1
\end{array}\right)
$$

distinct partitions of $N$ into $n p$ ordered nonnegative integral summands $m_{i, k}$ 's.

\footnotetext{
${ }^{3}$ Fix a Shellsort algorithm. Code the lengths of the inversion insertion paths in appropriate fixed order. Since the input permutation can be reconstructed from the coding, the overall length of the code must exceed the length of the shortest description of the input permutation. Since the latter is assumed to be incompressible this gives a lower bound on the sum total of the lengths of insertion paths and hence on the running time.
} 
Consider a subset $S$ of $n ! / 2^{n}$ input permutations, and let the maximum number of inversions among them be $M$. Clearly, $M>0$. Then, overestimating the number of partitions involved, $\sum_{N=0}^{M} D(N) \geq n ! / 2^{n}$ which implies $M D(M) \geq n ! / 2^{n}$. Then,

$$
\log (M D(M)) \geq(\log n !)-n .
$$

We know that $M \leq p n^{2}$ since every $m_{i, k} \leq n$. We have assumed $p<n$. Hence, $\log M<3 \log n$. The standard estimate gives $\log n !=n \log n-O(n)$ for $n \rightarrow \infty$. Estimate $\log D(M)$ by ${ }^{4}$

$$
\log \left(\begin{array}{c}
M+n p-1 \\
n p-1
\end{array}\right)=(n p-1) \log \frac{M+n p-1}{n p-1}+M \log \frac{M+n p-1}{M}+\frac{1}{2} \log \frac{M+n p-1}{(n p-1) M}+O(1) .
$$

The second term in the right-hand side is bounded as ${ }^{5}$

$$
\log \left(1+\frac{n p-1}{M}\right)^{M}<\log e^{n p-1}
$$

for all positive $M$ and $n p-1>0$. Since $0<p<n$ and $1 \leq M \leq p n^{2}$,

$$
\frac{1}{2(n p-1)} \log \frac{M+n p-1}{(n p-1) M} \rightarrow 0
$$

for $n \rightarrow \infty$. Therefore, $\log D(M)$ is majorized asymptotically by

$$
A=(n p-1)\left(\log \left(\frac{M}{n p-1}+1\right)+\log e\right)
$$

for $n \rightarrow \infty$. Altogether, $A+\log M \geq n \log n-O(n)$. With $p \leq(\epsilon / \log e) \log n(0<\epsilon<1)$, this can be rewritten as

$$
(n p-1) \log \left(\frac{M}{n p-1}+1\right) \geq(1-\epsilon) n \log n-O(n),
$$

and further as

$$
\log \left(\frac{M}{n p-1}+1\right) \geq\left(\frac{1-\epsilon}{p}\right) \log n-O\left(\frac{1}{p}\right) .
$$

The righthand side is positive and asymptotic to the first term for $n \rightarrow \infty$. Hence,

$$
M=\Omega\left(p n^{1+(1-\epsilon) / p}\right) .
$$

Theorem 2 The average computation time (number of inversions, for $p=o(\log n)$, and comparisons, for $n / 2 \geq p=\Omega(\log n))$ in $p$-pass Shellsort on lists of $n$ keys is at least $\Omega\left(p n^{1+1 / p}\right)$ for every increment sequence. The average is taken with all lists of $n$ items equally likely (uniform distribution).

Proof. Assume the terminology above. Take $S$ to be the special set of $n ! / 2^{n}$ input permutations using the least number of inversions. Then, the number of inversions made by algorithm $A$ for every permutation not in $S$ is at least $M$ in the previous proof. The theorem follows, since for $p=o(\log n)(\epsilon(n) \rightarrow 0$ for $n \rightarrow \infty$ in Theorem 1) the lower bound on the expected number of inversions of the sorting procedure is at least

$$
\left(1-\frac{1}{2^{n}}\right) \Omega\left(p n^{1+1 / p}\right)+\frac{1}{2^{n}} \Omega(0)=\Omega\left(p n^{1+1 / p}\right) ;
$$

and for $p=\Omega(\log n)$, the trivial lower bound on the number of comparisons is vacuously $p n=\Omega\left(p n^{1+1 / p}\right)$.

Our lower bound on the average-case can be compared with the Plaxton-Poonen-Suel $\Omega\left(n^{1+\epsilon / \sqrt{p}}\right)$ worst case lower bound [8]. Some special cases of the lower bound on the average-case complexity are:

\footnotetext{
${ }^{4}$ Use the following formula ([5], p. 10),

$$
\log \left(\begin{array}{l}
a \\
b
\end{array}\right)=b \log \frac{a}{b}+(a-b) \log \frac{a}{a-b}+\frac{1}{2} \log \frac{a}{b(a-b)}+O(1) .
$$

${ }^{5}$ Use $e^{a}>\left(1+\frac{a}{b}\right)^{b}$ for all $a>0$ and positive integer $b$.
} 
1. For $p=1$ our lower bound is asymptotically tight: it is the average number of inversions for Insertion Sort.

2. For $p=2$, Shellsort requires $\Omega\left(n^{3 / 2}\right)$ inversions (the tight bound is known to be $\Theta\left(n^{5 / 3}\right)[4]$ );

3. For $p=3$, Shellsort requires $\Omega\left(n^{4 / 3}\right)$ inversions (the best known upper bound is $O\left(n^{23 / 15}\right)$ in [2]);

4. For $p=\log n / \log \log n$, Shellsort requires $\Omega\left(n \log ^{2} n / \log \log n\right)$ inversions;

5. For $p=\log n$, Shellsort requires $p n=\Omega(n \log n)$ comparisons. When we consider comparisons, this is of course the lower bound of average number of comparisons for every sorting algorithm.

6. In general, for $n / 2 \geq p=p(n) \geq \log n$, Shellsort requires $\Omega(n \cdot p(n))$ comparisons (it requires that many comparisons anyway since every pass trivially makes about $n$ comparisons).

In [11] it is mentioned that the existence of an increment sequence yielding an average $O(n \log n)$ Shellsort has been open for 30 years. The above lower bound on the average shows that the number $p$ of passes of such an increment sequence (if it exists) is precisely $p=\Theta(\log n)$; all the other possibilities are ruled out.

\section{Conclusion}

The average-case performance of Shellsort has been one of the most fundamental and interesting open problems in the area of algorithm analysis. The simple average-case analysis of Insertion Sort (1-pass Shellsort), and similar analyses of Bubble sort, stack-sort and queue-sort are given in the preliminary version of this paper [6] and serve as further examples to demonstrate the generality and simplicity of our technique in analyzing sorting algorithms in general. Some open questions are:

1. Tighten the average-case lower bound for Shellsort. Our bound is not tight for $p=2$ passes.

2. Is there an increment sequence for $\log n$-pass Shellsort so that it runs in average-case $\Theta(n \log n)$ ?

\section{Acknowledgements}

We thank Don Knuth, Ian Munro, Vaughan Pratt, and Osamu Watanabe for discussions and references on Shellsort.

\section{References}

[1] J. Incerpi and R. Sedgewick, Improved upper bounds on Shellsort, Journal of Computer and System Sciences, 31(1985), 210-224.

[2] S. Janson and D.E. Knuth, Shellsort with three increments, Random Struct. Alg., 10(1997), 125-142.

[3] A.N. Kolmogorov, Three approaches to the quantitative definition of information. Problems Inform. Transmission, 1:1(1965), 1-7.

[4] D.E. Knuth, The Art of Computer Programming, Vol.3: Sorting and Searching, Addison-Wesley, 1973 (1st Edition), 1998 (2nd Edition).

[5] M. Li and P.M.B. Vitányi, An Introduction to Kolmogorov Complexity and its Applications, SpringerVerlag, New York, 2nd Edition, 1997.

[6] T. Jiang, M. Li, and P. Vitányi, The average-case complexity of Shellsort, Preliminary version, pp. 453-462 in the Proceedings of ICALP'99, LNCS 1644, Springer-Verlag, Berlin, 1999.

[7] A. Papernov and G. Stasevich, A method for information sorting in computer memories, Problems Inform. Transmission, 1:3(1965), 63-75. 
[8] C.G. Plaxton, B. Poonen and T. Suel, Improved lower bounds for Shellsort, Proc. 33rd IEEE Symp. Foundat. Comput. Sci., pp. 226-235, 1992.

[9] V.R. Pratt, Shellsort and Sorting Networks, Ph.D. Thesis, Stanford Univ., 1972.

[10] R. Sedgewick, Analysis of Shellsort and related algorithms, pp. 1-11 in: Proc. 4th Annual European Symposium on Algorithms, LNCS 1136, Springer-Verlag, 1996.

[11] R. Sedgewick, Open problems in the analysis of sorting and searching algorithms, Presented at Workshop on Prob. Analysis of Algorithms, Princeton, 1997. http://www.cs.princeton/ rs

[12] D.L. Shell, A high-speed sorting procedure, Commun. ACM, 2:7(1959), 30-32.

[13] A.C.C. Yao, An analysis of $(h, k, 1)$-Shellsort, J. of Algorithms, 1(1980), 14-50. 